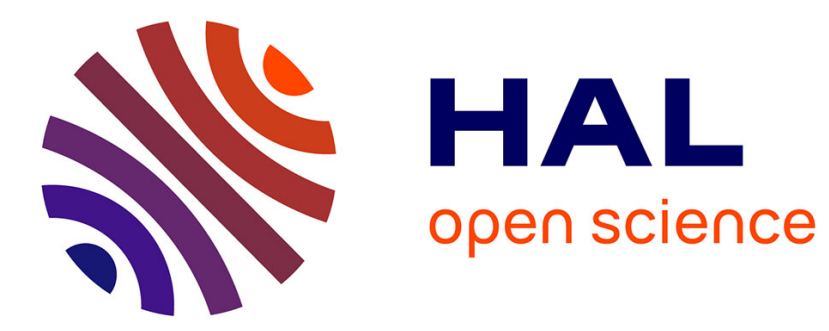

\title{
Fluctuation driven fixation of cooperative behavior
}

\author{
Bahram Houchmandzadeh
}

\section{To cite this version:}

Bahram Houchmandzadeh. Fluctuation driven fixation of cooperative behavior. BioSystems, 2015, 127, pp.60. 10.1016/j.biosystems.2014.11.006 . hal-00959575v2

\section{HAL Id: hal-00959575 \\ https://hal.science/hal-00959575v2}

Submitted on 13 Nov 2014

HAL is a multi-disciplinary open access archive for the deposit and dissemination of scientific research documents, whether they are published or not. The documents may come from teaching and research institutions in France or abroad, or from public or private research centers.
L'archive ouverte pluridisciplinaire HAL, est destinée au dépôt et à la diffusion de documents scientifiques de niveau recherche, publiés ou non, émanant des établissements d'enseignement et de recherche français ou étrangers, des laboratoires publics ou privés. 


\title{
Fluctuation driven fixation of cooperative behavior
}

\author{
Bahram Houchmandzadeh \\ Univ. Grenoble 1/CNRS, LIPhy UMR5588, Grenoble F-38401, France.
}

\begin{abstract}
Cooperative behaviors are defined as the production of common goods benefitting all members of the community at the producer's cost. They could seem to be in contradiction with natural selection, as non-cooperators have an increased fitness compared to cooperators. Understanding the emergence of cooperation has necessitated the development of concepts and models (inclusive fitness, multilevel selection, ...) attributing deterministic advantages to this behavior. In contrast to these models, we show here that cooperative behaviors can emerge by taking into account only the stochastic nature of evolutionary dynamics: when cooperative behaviors increase the population size, they also increase the genetic drift against non-cooperators. Using the Wright-Fisher models of population genetics, we compute exactly this increased genetic drift and its consequences on the fixation probability of both types of individuals. This computation leads to a simple criterion: cooperative behavior dominates when the relative increase in population size caused by cooperators is higher than the selection pressure against them. This is a purely stochastic effect with no deterministic interpretation.
\end{abstract}

Keywords: Fixation probability, variable size population, altruism, non-deterministic

\section{Introduction.}

One form of cooperative behaviors can be defined as the production of a common good by an individual that benefits everybody in the community. Such behavior has a cost in terms of fitness, as the producer devotes part of its resources to this task. To early evolutionary biologists, cooperative behaviors seemed to be in contradiction with natural selection $[1,2]$ : since selection acts on individuals, a non-cooperator has a higher fitness than a cooperator and will always invade the community. Cooperative behaviors however, specially in microbial world, are widespread. A few examples of such behaviors are light production in Vibrio fisheri[3], siderophore production in Pseudomonas aeroguinosa[4, 5], stalk formation by Dictyostelium discoidum[6, 7], decreased virulence in many pathogen-host systems[8]. All these cases are examples of a production of common good by an individual benefiting every individual in the 
community. More generally, these behaviors can be seen as particular cases of Niche Construction[9].

Researchers have investigated the deterministic advantages that these kinds of behaviors could confer on individuals. The major schools along this line of investigation are inclusive fitness[10, 11, 12] and multilevel selection[13, 14, 15, 16] and their associated variants[17], although the relative merits of these concepts are sometimes hotly debated $[18,19,20]$. These models have been extended to take into account finite size populations and stochastic effects. However, the emergence of cooperative behavior in these model is due to deterministic advantages (see the Discussion section). Throughout this article, "deterministic advantages" refers to factors which affects the fitness of an individual ; "deterministic formulation" refers to models where fluctuations are neglected and a simple differential equation (such as 1) is used to describe the dynamics of the population.

The aim of this article is not to discuss the relevance of these models, which have been documented in a large number of books and articles. The fact that cooperative behaviors are so widespread, however, behooves us to search for simple mechanisms to explain their emergence. I intend in this article to show that cooperative behaviors, by the simple act of increasing the population size, give an advantage to cooperators. The origin of this advantage is not deterministic, but has to be sought in the stochasticity of evolutionary dynamics. Note that the counterintuitive effect of stochasticity (favoring the a priori disadvantaged type) has been shown in spatially extended populations [21, 22, 23, 24], where space is supposed to play the crucial role [21]. We show in this paper that this phenomenon can take place even in well mixed populations and space plays then the role of an amplification factor (see Discussion section).

Evolution is an interplay between deterministic causes broadly called fitness, and random events such as sampling between generations. An advantageous mutant does not spread with certainty but has only a greater probability of invading the community and of being fixed. This probability, called the fixation probability, is the relevant quantity to weight deterministic versus stochastic causes[25].

Consider an asexual population of fixed size $N$, with two types of individuals $A$ and $S$, where $S$ types have a constant positive excess relative fitness $s$ compared to $A$. The deterministic differential equation describing the variation of the proportion $x=N_{A} / N$ of the $A$ type is[26]:

$$
d x / d t=-s x(1-x)
$$

and leads to the disappearance of $A$ individuals $(x \rightarrow 0)$.

Going beyond the deterministic approach, one can solve the full stochastic dynamics of such a model and extract the invasion capacity of these two types, i.e. the fixation probability $\pi_{1}^{i}$ of one individual of type $i$ introduced into a population consisting entirely of the other type. In the framework of the WrightFisher, for a population of fixed size $N$, in the small selection pressure limit 
$N s \ll 1$ ( see [26] and the exact derivations in equation (10) ):

$$
\begin{aligned}
& \pi_{1}^{A}=\frac{1}{N}-s \\
& \pi_{1}^{S}=\frac{1}{N}+s
\end{aligned}
$$

Therefore, if $s>0$ then $\pi_{1}^{S}>\pi_{1}^{A}$ and type $S$ individuals have a higher invasion capacity than type $A$ individuals. In this case, the ratio of invasion capacities has the same information content as the deterministic approach: both lead to the conclusion that $s>0$ favors the $S$ type. The equivalence between these two approaches has led researchers to investigate the existence of deterministic advantages that could favor the cooperators ( $A$ individuals) against noncooperators ( $S$ individuals) even though $s$, the bare fitness of $S$ (or equivalently, the cost of altruism to $A$ ) is positive.

Fluctuations and random events can however be more subtle and alter the equivalence between deterministic and stochastic modeling. In particular, we can have $\pi_{1}^{S}<\pi_{1}^{A}$ even when $s>0$, without any hidden deterministic advantage. This is the case of a cooperative behavior that increases the population size.

Biologically, the dependence of population size on the number of cooperators happens for example in situations where individuals compete for limited resources and two strategies are possible: an economic use of the resources leading to high population size but low growth rate or a dispendious use of resources leading to high growth rate but low population size (known as "the tragedy of the common"[27]). In the microbial word, this phenomenon (called high yield versus high rate) is common where individuals can have two strategies, for example for ATP synthesis (respiration versus fermentation in yeast)[28, 29, 30] and competition in biofilms where a high yield mutant give rise to larger biofilm, even though it has a lower growth rate [31]. Rainey and Rainey[32] have studied another case, where the production of a polymeric molecule by the cooperator type leads to large decrease in growth rate but higher densities.

A similar observation was performed by Chuang et al. in an engineered microbial system [33] where two type of bacteria were competing in an antibiotic containing media. One type (called producers, i.e. cooperators, A ) was designed to produce an anti-biotic resistance molecule ; the resources used to produce this molecule would reduce the growth rate (fitness) of this type. The other type of bacteria (called non producers, i.e. non cooperators, $S$ ) was designed not to produce the antibiotic resistance molecule and would therefore have a higher growth rate compared to producers. The common good in this case is the anti-biotic resistance molecule. In a culture composed only of the $S$ type, no common good is available and the population reaches a low density. In a population composed only of $A$ type, the common good is abundant and the population reaches a high density. In a mixed population, the final population density would reach an intermediate level. However, because non producers have a higher relative growth rate, they always increase their proportion during one growth cycle. 

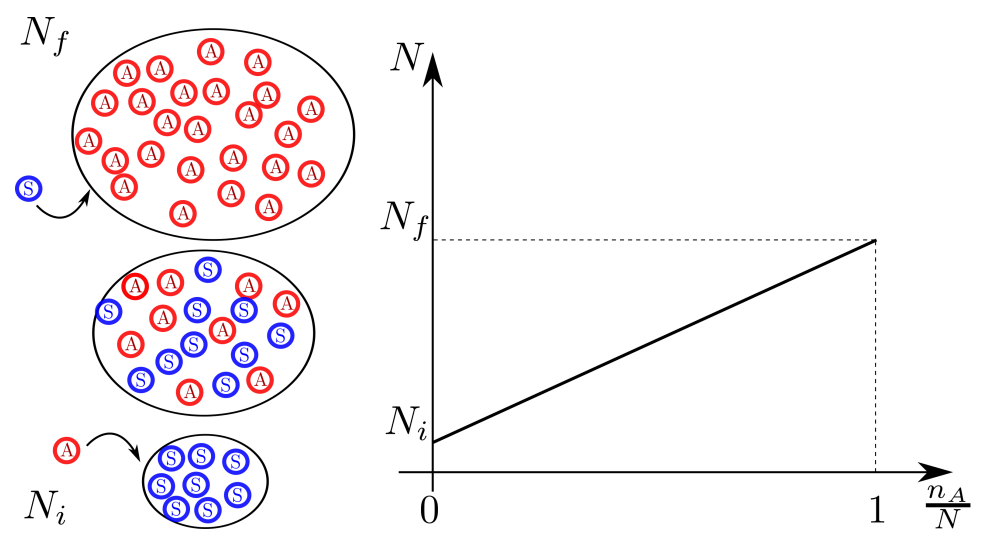

Figure 1: (Color online) Scheme of a cooperative behavior where the population size $N$ of the habitat is an increasing function of the proportion $x$ of type $A$ individuals : $N=N(x)$. For a habitat formed of only $S$ type individuals, $N(0)=N_{i}$. When only $A$ individuals are present, $N(1)=N_{f}$, where $N_{f}>N_{i}$. The invasion capacity of each type is defined as the fixation probability of one $i$ type introduced into a community formed only of type $j$.

Consider a system where the population size is a function of the proportion of cooperators, varying between $N_{i}$ when only $S$ type is present and $N_{f}$ where only $A$ type is present, with $N_{i}<N_{f}$ (figure 1). We suppose that $S$ types have a constant excess relative fitness $s>0$. The deterministic equation (1) does not change and will again lead to the $A$ 's extinction. The stochastic dynamic however can be different. When one $A$ mutant is introduced in a population of $S$, the initial population size is $N_{i}$; on the other hand, when one $S$ type is introduced in a population of $A$, the initial population size is $N_{f}$. Using expressions $(2,3)$ as a back of the envelope estimation of the invasion capacity of both types therefore yields (an exact derivation is given in the following section):

$$
\begin{aligned}
& \pi_{1}^{A}=\frac{1}{N_{i}}-s \\
& \pi_{1}^{S}=\frac{1}{N_{f}}+s
\end{aligned}
$$

We observe that we can have $\pi_{1}^{S}<\pi_{1}^{A}$ even though $s>0$, if

$$
2 s<\frac{1}{N_{i}}-\frac{1}{N_{f}}
$$

If the selection pressure against cooperators is smaller than the relative variation in the population size due to cooperators, then the latter type is favored and has a higher invasion capacity. This is a purely stochastic effect with no deterministic counterpart and is due to the fact that cooperators increase the genetic drift of non-cooperators.

We had previously shown the existence of this effect using a two dimensional Markov chain approach of a generalized Moran model[34]. This approach 
however is mathematically intricate and only approximate solutions could be obtained at small selection pressure. The effect however can be understood in a much simpler way using a classical Wright-Fisher (WF) model of population genetics, which I develop in the following sections, where very general results can be obtained. The WF model is a well studied generic model of population genetics, shown to be equivalent to many other models of population genetics[35].

The article is organized as follow. In the Theory section, I recall the key points of the classical WF model. In the Result section, first a simple system is considered where the population size is a linear function of the proportion of cooperators. An exact solution for the fixation probabilities is then obtained and it is shown that cooperators can have a higher invasion capacities than non-cooperators, even when the cost of cooperation is always positive. The next subsection generalizes this concept to arbitrary dependence of the population size on the proportion of cooperators; a very simple and general criterion is then obtained for cooperators to prevail. Finally, the extension to diploid populations is considered in the following subsection. The conclusion section put these results into perspective in respect to other models of the emergence of cooperation.

\section{Methods.}

The stochastic simulations follow precisely the described model (eq.14). (i) Given two numbers $n_{A}$ and $n_{S}$ representing the number of $A$ and $S$ at a given generation, the program computes $x=n_{A} /\left(n_{A}+n_{S}\right), u=u(x)$ according to equation (6) and $N=N(x)$ according to equation (16). A binomial random number generator is then used to find $n_{A}^{\prime}$ and $n_{S}^{\prime}=N-n_{A}^{\prime}$ in the next generation according to equation (14). (ii) Beginning with a given number of $n_{A}$ and $n_{S}$ at first generation, the program generates one random path by iterating the above process until either $n_{A}$ or $n_{S}$ reaches zero. (iii) $M$ such paths are generated and the fixation probability $\pi(x)$ of $A$ individuals is computed from the proportion of paths for which $n_{S}=0$. Usually, $M=10^{8}$ paths are used to compute the fixation probability for a given $x$. (iv) The initial proportion $x$ is varied between 0 to 1 to compute the function $\pi(x)$. The numerical simulation, written in $\mathrm{C}++$, is performed on a 64-core computer.

\section{Theory.}

I recall the main results of the classical Wright-Fisher model for the sake of clarity of the following sections. In a community of fixed size $N$, two types of asexual adult individuals $A$ and $S$ of abundance $n_{A}$ and $n_{S}\left(n_{A}+n_{S}=N\right)$ produce progeny. This progeny is then sampled to form the next generation of adults. The sampling process is biased toward the $S$ type which has an excess relative fitness $s$ which we suppose to be small $(0<s \ll 1)$. 
The transition probability to have $k$ individuals of type $A$ in the next generation $G_{i+1}$ when $n_{A}$ individuals were present at generation $G_{i}$ is binomial $[36,26]$ :

$$
P\left(k \mid n_{A}\right)=\left(\begin{array}{c}
N \\
k
\end{array}\right) u^{k}(1-u)^{N-k}
$$

where

$$
\begin{aligned}
u & =\frac{x}{x+(1+s)(1-x)} \\
& =x-s x(1-x)+\mathcal{O}\left(s^{2}\right)
\end{aligned}
$$

In the above expression, $x=n_{A} / N$ designates the proportion of $A$ in $G_{i}$. The bias $s$ toward the selection of one type can be due to the increase in mean number of progeny, the variability in their production[37, 35], or any other similar phenomena.

The exact dynamics of the above stochastic process is not known, but one can resort to the diffusion approximation $[38,26]$ to compute various quantities of interest. This computation is based on the change in the mean and variance of the proportion of $A$ types in the next generation, which, to the first order in $s$ is:

$$
\begin{aligned}
a(x) & =\langle y \mid x\rangle-x=-s x(1-x) \\
b(x) & =\frac{1}{2} \operatorname{Var}(y \mid x)=\frac{1}{2 N} x(1-x)
\end{aligned}
$$

where $y$ is the proportion of $A$ in the next generation; $\langle y \mid x\rangle$ designates the expectation of $y$ conditioned on $x$, the proportion of $A$ in the present generation. The fixation probability $\pi(x)$ of the $A$ type present with proportion $x$ at the first generation can be computed from the backward diffusion approximation of stochastic dynamics[38] :

$$
a(x) \frac{d \pi(x)}{d x}+b(x) \frac{d^{2} \pi(x)}{d x^{2}}=0
$$

As $-a(x) / b(x)=2 N s$, the use of boundary conditions $\pi(0)=0, \pi(1)=1$ leads to the well known Kimura solution:

$$
\begin{aligned}
\pi(x) & =\frac{1-e^{2 N s x}}{1-e^{2 N s}} \\
& \approx x-N s x(1-x) \text { for } N s \ll 1
\end{aligned}
$$

The invasion capacity of both types are readily obtained from the above expression

$$
\pi_{1}^{A}=\pi(1 / N) ; \pi_{1}^{S}=1-\pi(1-1 / N)
$$

and are equal to expressions $(2,3)$ in the small selection pressure limit $N s \ll 1$. The ratio of invasion capacities reads

$$
R_{S A}=\frac{\pi_{1}^{S}}{\pi_{1}^{A}}=\frac{1+N s}{1-N s} \approx 1+2 N s
$$




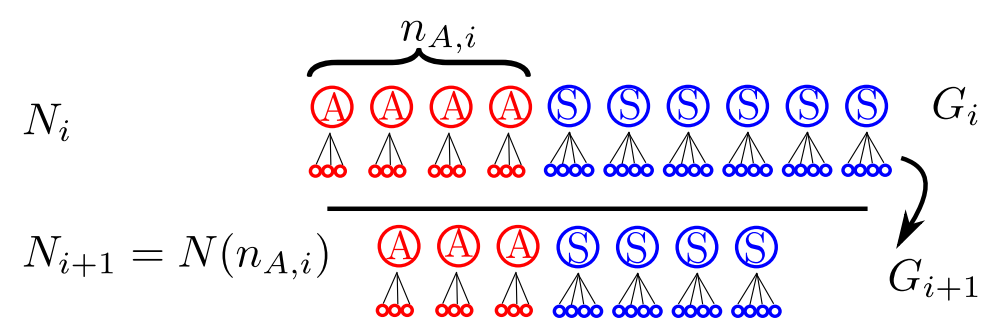

Figure 2: (Color online) Scheme of sampling between generations in the present model : The population size $N_{i+1}$ of generation $G_{i+1}$ is a function of the number of cooperators $n_{A, i}$ present at generation $G_{i}: N_{i+1}=N\left(n_{A}\right)$. The selection process consists in selecting $N_{i+1}$ individuals among the progeny at $G_{i}$.

and $R_{S A}>1$ if $s>0$. Note that here we use $s$ as the relative excess advantage of the $S$ type, or equivalently, the cost of $A$ type. Hence the change in the sign of $s$ in expression (11) compared to similar expressions used in the literature. The reason behind this choice is that in the following, $A$ will designates the cooperators with a positive cost for cooperation.

\section{Results.}

\subsection{Variable size population.}

Consider now a system in which the population size is not constant, but is an increasing function of the number of cooperators $n_{A}$ (figure 1). The stochastic behavior of such a system can be modelled as follow: as in the fixed size habitat before, both $A$ and $S$ types at generation $G_{i}$ produce progeny ; however, the population size $N_{i+1}$ of the next generation $G_{i+1}$ depends on the number (or proportion) of cooperators in $G_{i}$. Hence $N_{i+1}=N\left(n_{A, i}\right)$ individuals among the progeny are randomly selected to form the next generation (figure 2). The probability of having $k$ individuals of type $A$ in generation $G_{i+1}$, knowing that there were $n_{A, i}$ individuals at generation $G_{i}$ is binomial :

$$
P\left(k \mid n_{A, i}\right)=\left(\begin{array}{c}
N_{i+1} \\
k
\end{array}\right) u^{k}(1-u)^{N_{i+1}-k}
$$

where $u$ has the same definition as in (6). We can repeat all the arguments for the computation of relative change in the mean and variance of the proportion $x=n_{A} / N$ of $A$ types, keeping in mind that the only difference in the present model is that $N=N(x)$ is no longer constant. In particular, the fixation probabilities are given by the same backward diffusion equation

$$
a(x) \frac{d \pi(x)}{d x}+b(x) \frac{d^{2} \pi(x)}{d x^{2}}=0
$$

where this time,

$$
-\frac{a(x)}{b(x)}=2 s N(x)
$$


For the sake of simplicity, in this subsection we suppose a linear form for $N(x)$ (figure 1):

$$
N(x)=(1-x) N_{i}+x N_{f}
$$

where $N_{f}$ and $N_{i}\left(N_{i}<N_{f}\right)$ are the carrying capacities of the habitat when composed only of $A$ types and $S$ type. The next subsection generalizes the computation to arbitrary form of $N(x)$.

Let us first consider the trivial case of $s=0$, i.e. a cooperative behavior which increases the population size without any cost to $A$, the producers of the common good. In this case, $a(x)=0$ and the solution of eq.(15), taking into account the boundary conditions is given by $\pi(x)=x$. The invasion capacities of $A$ and $S$ is therefore

$$
\begin{aligned}
& \pi_{1}^{A}=\pi\left(x=1 / N_{i}\right)=1 / N_{i} \\
& \pi_{1}^{S}=1-\pi\left(x=1-1 / N_{f}\right)=1 / N_{f}
\end{aligned}
$$

and $\pi_{1}^{A}>\pi_{1}^{S}$. This case illustrates the clear advantage of the cooperator type $A$ and the stochastic cost to non-cooperators in the form of increased genetic drift. Note that the deterministic equation $d x / d t=a(x)$ cannot predict this outcome.

When $s>0$, the stochastic advantage of $A$ types is reduced, but can persist up to some value $s$ max which we compute below. The differential equation (15) can still be easily solved. Let us express $N_{i}$ and $N_{f}$ in terms of their mean and relative difference

$$
\begin{aligned}
\bar{N} & =\left(N_{f}+N_{i}\right) / 2 \\
2 \delta & =\left(N_{f}-N_{i}\right) / \bar{N}
\end{aligned}
$$

Then, setting

$$
\psi(x)=2 \bar{N} s x(1-\delta+\delta x)
$$

we have

$$
\frac{d \pi}{d x}=C \exp (\psi(x))
$$

Integrating once more and taking into account the boundary conditions $\pi^{A}(0)=$ $0, \pi^{A}(1)=1$, the solution can be written as

$$
\pi(x)=\frac{f(0)-f(x)}{f(0)-f(1)}
$$

where

$$
f(x)=\frac{e^{2 \bar{N} s\left((1-\delta) x+\delta x^{2}\right)}}{\sqrt{2 \bar{N} s \delta}} D\left(\sqrt{\frac{\bar{N} s}{2 \delta}}(1-\delta+2 \delta x)\right)
$$

and

$$
D(z)=e^{-z^{2}} \int_{0}^{z} e^{u^{2}} d u
$$




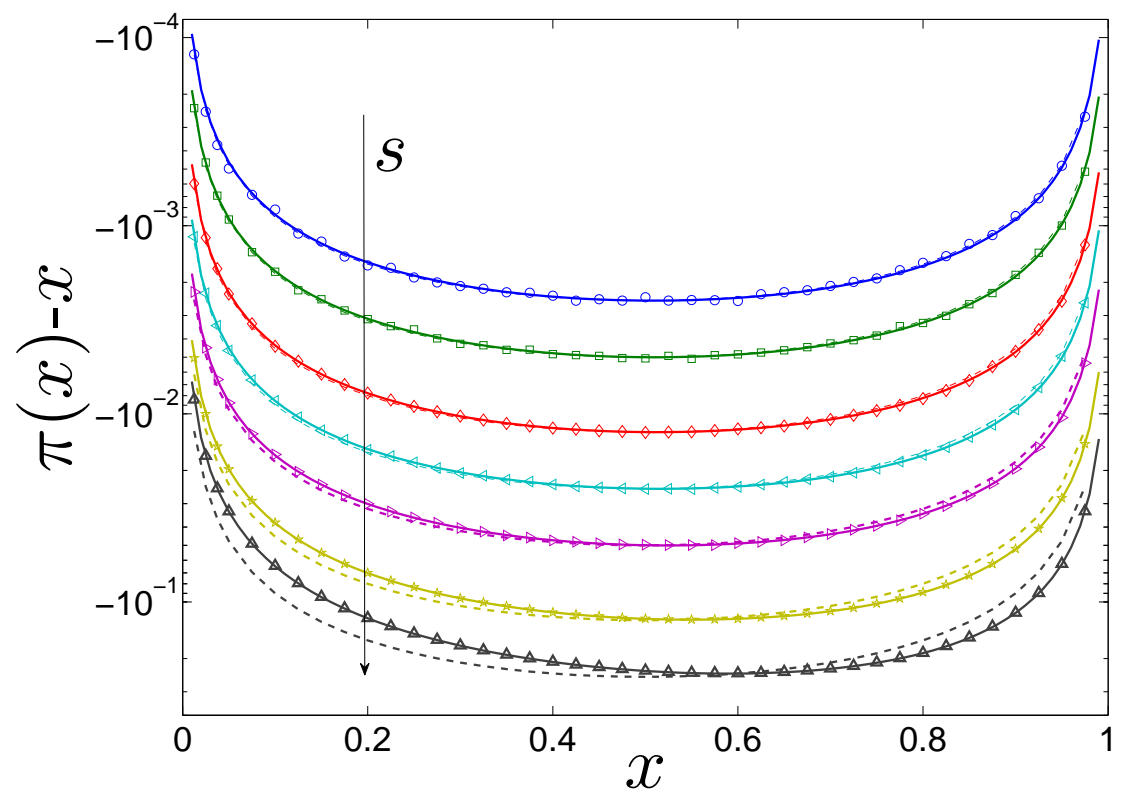

Figure 3: (Color online) Numerical computation of $\pi(x)$ and its comparison with theoretical values for $N_{i}=90, N_{f}=110$ and $s=(1,2,5,10,20,50,100) \times 10^{-4}$. The arrow indicates the direction of increasing $s$. For each value of initial proportion of $A$ individuals $x \in[0,1]$, a random discrete path is generated by determining the population size in the next generation according to relation (16) and then sampling of the present generation to constitute the next generation according to relation (14); the process is stopped by the loss of either $A$ or $S$ individuals. $10^{8}$ such paths are generated to compute, for each initial proportion $x$, its fixation probability $\pi(x)$. Symbols : values obtained from numerical simulations ; solid lines : theoretical values according to expression (20); dashed lines : small selection pressure approximation (eq. 21).

is the Dawson function. Note that for $\delta \rightarrow 0$; the above expression for fixation probability converges to the expression (11) for fixed population size. In the case of small selection pressure against $A(\bar{N} s \ll 1)$ and small relative change in the size of the habitat $\delta \ll 1$, expanding the fixation probability (20) to the second order in $\bar{N} s$ and $\delta$, we find

$$
\pi(x) \approx x-\bar{N} s x(1-x)+\frac{\bar{N} s}{3}(\bar{N} s+\delta) x(1-x)(1-2 x) .
$$

Note that this limit could have been obtained by direct integration of the power series expansion of $\exp (\psi(x))$ in expression (19). Direct integration of power series allows more complicated laws of population size $N(x)$ to be taken into account, when exact solutions are no longer available.

Figure 3 shows the excellent agreement between numerical simulations of the above stochastic process and the theoretical predictions (eqs. 20,21). 
Let us now compute the invasion capacity of each type by keeping only the first order terms:

$$
\begin{aligned}
& \pi_{1}^{A}=\pi\left(\frac{1}{N_{i}}\right) \approx \frac{1}{N_{i}}-\frac{\bar{N}}{N_{i}} s \\
& \pi_{1}^{S}=1-\pi\left(1-\frac{1}{N_{f}}\right) \approx \frac{1}{N_{f}}+\frac{\bar{N}}{N_{f}} s
\end{aligned}
$$

The ratio of invasion capacities now becomes:

$$
\begin{aligned}
R_{S A} & =\frac{\pi_{1}^{S}}{\pi_{1}^{A}}=\left(\frac{1-\delta}{1+\delta}\right)\left(\frac{1+\bar{N} s}{1-\bar{N} s}\right) \\
& \approx 1+2 \bar{N} s-2 \delta
\end{aligned}
$$

We see that, contrary to the fixed population size case (eq. 13), we can have $R_{S A}<1$ even when $s>0$ ! The criterion for cooperators to prevail is simply

$$
\delta>\bar{N} s
$$

Figure 4 shows the excellent agreement between $R_{S A}$ obtained from numerical results and the theoretical prediction (eq. 20). Note that for large $\delta$, the criterion (24) underestimates the advantage of $A$ individuals, which can prevail at a higher cost of cooperation. The general form of the criterion is given in the next subsection

Let us stress again that the $A$ type advantage is due purely to the stochasticity of natural selection and has no deterministic interpretation. It is due to the interplay between genetic drift and deterministic effect. The deterministic equation, neglecting fluctuations, is as before

$$
\frac{d x}{d t}=a(x)=-s x(1-x)
$$

and predicts the disappearance of $A$ individuals that have a negative relative excess fitness.

\subsection{Solution for general form of $N(x)$ and $s(x)$.}

The conditions necessary for $R_{S A}<1$ can be obtained for any form of $N(x)$ without computing $\pi(x)$ explicitly. First, note that the first integral of the Kimura equation (15) is

$$
\begin{aligned}
\log \left(\frac{\pi^{\prime}(x)}{\pi^{\prime}(0)}\right) & =-\int_{0}^{x} \frac{a(u)}{b(u)} d u \\
& =2 s \int_{0}^{x} N(u) d u
\end{aligned}
$$

The invasion capacities can be expressed in terms of the function $\pi^{\prime}(x)$ :

$$
\begin{aligned}
& \pi_{1}^{A}=\pi\left(1 / N_{i}\right)=\pi^{\prime}(0)\left(1 / N_{i}\right)+\mathcal{O}\left(1 / N_{i}^{2}\right) \\
& \pi_{1}^{S}=1-\pi\left(1-1 / N_{f}\right)=\pi^{\prime}(1)\left(1 / N_{f}\right)+\mathcal{O}\left(1 / N_{f}^{2}\right)
\end{aligned}
$$



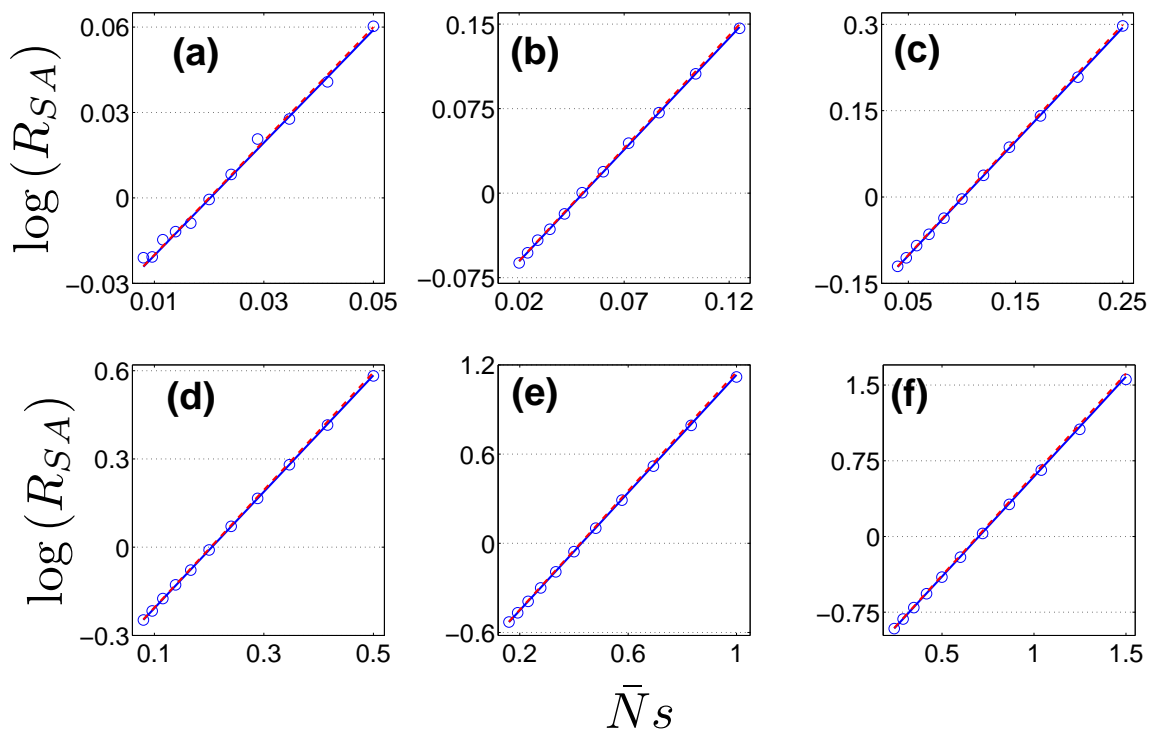

Figure 4: (Color online) Logarithm of the ratio of invasion capacities $R_{S A}=\pi_{1}^{S} / \pi_{1}^{A}$ as a function of selection pressure $\bar{N} s$ for $\bar{N}=100$ and increasing value of $\delta=0.02,0.05,0.1,0.2,0.4,0.6$ (a-f). Symbols : fixation probability ratio obtained from numerical simulations as detailed in figure 3; solid lines : theoretical predictions of fixation probabilities obtained from eq. (20) ; dashed lines : general solution given by relation (25). 
because of the boundary conditions $\pi(0)=0$ and $\pi(1)=1$. Therefore, to the first order in $1 / N$, the ratio of invasion capacities is:

$$
R_{S A}=\frac{\pi_{1}^{S}}{\pi_{1}^{A}}=\frac{\pi^{\prime}(1)}{\pi^{\prime}(0)} \frac{N_{i}}{N_{f}}
$$

and therefore

$$
\log \left(R_{S A}\right)=2 s \int_{0}^{1} N(u) d u+\log \left(N_{i} / N_{f}\right)
$$

The condition for $A$ to prevail,i.e. $R_{S A}<1$, is then equivalent to

$$
2 s \int_{0}^{1} N(u) d u<\log \left(N_{f} / N_{i}\right)
$$

For the simple case investigated in the preceding subsection, in which $N(x)=$ $\bar{N}(1-\delta+2 \delta x)$ the left hand side of relation $(26)$ evaluates to $2 \bar{N} s$ and the criterion becomes

$$
2 \bar{N} s<\log \left(\frac{1+\delta}{1-\delta}\right)=\log \left(\frac{N_{f}}{N_{i}}\right)
$$

which reduces to expression $(24)$ for small $\delta$, as $\log [(1+\delta) /(1-\delta)] \approx 2 \delta$. The above criterion states again that when the selection pressure against the cooperators is less than the relative change in population size, cooperators are favored.

The accuracy of this criterion, for the case of linear $N(x)$, is shown in figure 4. The general criterion (26) is not limited to small selection pressure or small variation in population size. The condition $N \gg 1$ is still necessary for the validity of the diffusion approximation[39].

The present article was restricted until now to the case where the cost of altruism $s$ was constant. This is the case for example of light production in $V$. fisheri : bacteria engaging in light production decrease their growth rate, and non-cooperator mutants gain a fixed increased fitness compared to them[40]. In many situations however, this is a to restrictive hypothesis as the availability of the common good would in principle diminish the fitness difference between producers and non-producers of the common good. For example, when the cost of producing the common good for an individual is constant $(C)$ but the benefit received from others depend on the number of producers $B x$. The overall cost of altruism will then be $s(x)=C-B x$. Therefore, in general, the cost of altruism should be considered to depend on the proportion of altruists, i.e. $s=s(x)$, and the cost would be a decreasing function of the proportion of altruists. The precise measurement of $s(x)$ is best performed in experiments with bacterial colonies[33, 41, 29].

The restriction to constant $s$ can easily be relaxed and the above computations and the criteria (26) can be generalized to the case where $s=s(x)$. Repeating the above computations for this case, the criterion for $A$ to prevail becomes

$$
2 \int_{0}^{1} s(u) N(u) d u<\log \left(N_{f} / N_{i}\right)
$$


The case $s$ constant is the "worst case" scenario, showing that even in this case, altruists can emerge by simple genetic drift and varying population size. If $s(x)$ is a decreasing function of $x$, then obviously $\int_{0}^{1} s(u) N(u) d u<s(0) \int_{0}^{1} N(u) d u$ and the emergence of altruists is favored even more. In the specific case where $s(x)$ changes its sign (as it is supposed in many frequency-dependent models), altruists can emerge even for constant size populations $N_{i}=N_{f}=N$ if $\int_{0}^{1} s(u) d u<0$.

\subsection{Extension to diploid populations.}

The above results can be generalized to diploid, randomly mating populations when cooperative behavior is caused by a single gene. Consider a diploid population of size $N$ corresponding to $2 N$ gametes. The fitness of ( $A A, A S, S S)$ individuals will be denoted $(1,1+s(1 / 2-\epsilon), 1+s)$ where $s$, as before is the relative fitness value of the allele $S$ and $\epsilon \in[-1 / 2,1 / 2]$ is the dominance of allele $A ; \epsilon=0$ corresponds to no dominance. As before, $x$ will designate the frequency of allele $A$. Following the arguments of the previous sections, we can write the number of $A$ allele in the next generation as

$$
P\left(k \mid n_{A, i}\right)=\left(\begin{array}{c}
2 N_{i+1} \\
k
\end{array}\right) u^{k}(1-u)^{2 N_{i+1}-k}
$$

where $[26]$

$$
\begin{aligned}
u & =\frac{x^{2}+(1+s(1 / 2-\epsilon)) x(1-x)}{x^{2}+2(1+s(1 / 2-\epsilon)) x(1-x)+(1+s)(1-x)^{2}} \\
& =x-s x(1-x)(1+2 \epsilon-4 \epsilon x)+\mathcal{O}\left(s^{2}\right)
\end{aligned}
$$

For the population size, we will use a generalization of relation (16) :

$$
\frac{N^{2}}{\bar{N}^{2}}=(1-\delta+2 \delta x)^{2}+2 \epsilon \delta x(1-x)
$$

where $\bar{N}$ and $\delta$ were defined in $(17,18)$. This relation reduces to (16) when $\epsilon=0$ and ensures that $N=\bar{N}=$ const when $\delta=0$.

Repeating the computations of the previous section in the regime where $\bar{N} s \ll 1$ and $\delta \ll 1$, and keeping the lowest order terms leads to

$$
\pi(x)=x-2 \bar{N} s x(1-x)\left(1+\frac{2 \epsilon}{3}(1-2 x)\right)
$$

from which we can compute the ratio of invasion capacities $R_{S A}=\pi_{1}^{S} / \pi_{1}^{A}$ :

$$
\begin{aligned}
R_{S A} & =\left(\frac{1-\delta}{1+\delta}\right)\left(\frac{1+2 \bar{N} s(1-2 \epsilon / 3)}{1-2 \bar{N} s(1+2 \epsilon / 3)}\right) \\
& \approx 1+4 \bar{N} s-2 \delta
\end{aligned}
$$

The simplest diploid case (random mating, no linkage disequilibrium) is similar to the haploid case and the criterion for cooperators to prevail does not change. 


\section{Discussion and Conclusion.}

The problem of the emergence of cooperative behaviors and "altruism" has been a conundrum in evolutionary biology and has attracted a very large number of contributions from different fields. We have shown in this article that this conundrum may not exist at all, if we shift our attention from deterministic advantages to fluctuation induced advantages. The original Wright-Fisher model, developed in the 20's, clarified the concepts of stochasticity in population genetics and showed that a mutant, even when deleterious, has some probability of invading the community, i.e. $\pi_{1}^{A}>0$. We have, in this article, extended this concept by showing that it is even possible for the deleterious mutant to have a higher invasion capacity than the wild type, i.e. $\pi_{1}^{A}>\pi_{1}^{S}$. This is based on the fact that purely fluctuation induced advantages can overcome the disadvantages and the cost of cooperative behaviors if the relative increase in the population size induced by cooperators is higher than the cost of altruism. This demonstration has been achieved by the use of the generic Wright-Fisher model, which captures in very simple terms the combined effects of finite size populations and deterministic advantages.

All the existing models of cooperation (kin/multilevel/reciprocity/...) have also been extended to finite populations in order to take into account the importance of fluctuations. The important point to stress is that in these models, there are always deterministic advantages associated with cooperation. In other terms, the deterministic drift term $a(x)$ changes its sign for some $x \in] 0,1[$ and has more than two zeros. Therefore, the deterministic equation for the proportion of $A$,

$$
d x / d t=a(x)
$$

has more than one stable point, one of which corresponds to the dominance of $A$ types. For example, the replicator dynamics used in the context of evolutionary game theories uses[42, 43]

$$
a(x)=x(1-x)(A x+B(1-x))
$$

and for $A>0, B<0$ and $B /(B-A) \in[0,1]$, the dynamics possesses two stable points $x=0$ and $x=1$. Taking into account fluctuations and finite size populations then helps to explain how a single $A$ mutant can emerge and dominate the habitat [44, 45]. The same kind of arguments can be made for multilevel selection theories, where the deterministic models already explain the possibility for the existence of cooperators[15] and then the computation can be extended to take into account fluctuations[46].

Natural populations are geographically extended and the addition of space to the evolutionary dynamics introduces new features. Van Baleen and Rand [21], using a moment closure approximation for spatially extended population, have argued that altruistic behaviors can emerge due to the stochasticity of the evolutionary dynamics even when the deterministic equations predict their disappearance. The various extensions/generalizations of their work has been reviewed by Lehman and Rousset [47]. Perc[23] has considered the case of spatially extended population of defectors and cooperators in the context of game 
dynamics, and has shown that the addition of some amount of randomness to the payoff of different strategies can reverse the direction of the game: cooperators can reach a substantial proportion of the total population, even though their extinction is predicted from a deterministic formulation of the game. A similar phenomenon happens when the randomness in payoff is spatially distributed [24] and various extension of these models are reviewed in [48].

In the case of varying population size, the addition of space can have an amplifying effect on the stochastic advantage of the $A$ type discussed here. It can indeed by shown that at low migration rate, the invasion capacity of $S$ type mutant tend toward zero, while the invasion capacity of the $A$ type remain finite [34]. A similar result was obtained by Hallatschek[49] when investigating the dynamics of Fisher waves for varying population size. These results were obtained for populations subdivided into patches and exchanging migrants between them. An extension of the present work would be to derive these results for continuously distributed populations and obtain a consistent expression for the effective population size $\bar{N}_{e}$ of spatially structured populations in the context of cooperative behaviors.

To summarize, in the model we present in this article, there is no deterministic advantages associated with cooperations and $a(x)<0$ for all $x \in[0,1]$. The only driving force in the present model is provided by fluctuations due to finite size populations. The key point, which we have demonstrated in the preceding sections, is that the invasion capacity $\pi_{1}^{A}$ of the cooperators can be higher than the invasion capacity of defectors $\pi_{1}^{S}$, even when the cost of cooperation $s$ is always positive and the deterministic approach leads to the extinction of the $A$ types.

The aim of this article is not to contest the merits of existing models such as kin or multilevel selection, which have been investigated during the last forty years with a large number of case studies. I propose an alternative approach to the problem of cooperation, that is complimentary to the existing models and which restores the key ingredients of population genetics to this field.

Acknowledgment. . I am grateful to M. Vallade, E. Geissler and O. Rivoire for careful reading of the manuscript and fruitful discussions. The generalization of criteria (28) to the frequency-dependent selection was suggested by the anonymous referee. The author declares to have no conflict of interest.

[1] E. Sober, D. S. Wilson, Unto others: The Evolution and Psychology of Unselfish Behavior, Harvard university press, Cambridge, MA, 1999.

[2] L. A. Dugatkin, The Altruism Equation, Princeton University Press, Princeton, N.J., 2006.

[3] K. L. Visick, E. G. Ruby, Vibrio fischeri and its host: it takes two to tango., Curr Opin Microbiol 9 (2006) 632-8.

[4] S. A. West, A. Buckling, Cooperation, virulence and siderophore production in bacterial parasites., Proc R Soc Lond B Biol Sci 270 (2003) 37-44. 
[5] F. Harrison, A. Buckling, Cooperative production of siderophores by Pseudomonas aeruginosa., Front Biosci 14 (2009) 4113-26.

[6] R. H. Kessin, Dictyostelium: Evolution, Cell Biology and the Development of Multicellularity., Cambridge Univ. Press, Cambridge, 2001.

[7] K. R. Foster, G. Shaulsky, J. E. Strassmann, D. C. Queller, C. R. L. Thompson, Pleiotropy as a mechanism to stabilize cooperation, Nature 431 (2004) 693-696.

[8] S. P. Diggle, Microbial communication and virulence: lessons from evolutionary theory., Microbiology 156 (2010) 3503-12.

[9] J. Odling-smee, D. H. Erwin, E. P. Palkovacs, M. W. Feldman, Niche Construction Theory: a practical guide for ecologists., Q Rev Biol 88 (2013) $3-28$.

[10] W. D. Hamilton, The genetical evolution of social behaviour. I., J Theor Biol 7 (1964) 1-16.

[11] R. E. Michod, The Theory of Kin Selection, Annu Rev Ecol Syst 13 (1982) $23-55$.

[12] A. Gardner, S. A. West, G. Wild, The genetical theory of kin selection., J Evol Biol 24 (2011) 1020-43.

[13] R. C. Lewontin, The Units of Selection, Annu Rev Ecol Syst 1 (1970) 1-18.

[14] D. S. Wilson, The Group Selection Controversy: History and Current Status, Annu Rev Ecol Syst 14 (1983) 159-187.

[15] A. Silva, J. Fontanari, Deterministic group selection model for the evolution of altruism, The European Physical Journal B 7 (1999) 385-392.

[16] A. Traulsen, M. A. Nowak, Evolution of cooperation by multilevel selection., Proc Nat Acad Sci USA 103 (2006) 10952-5.

[17] M. A. Nowak, Five rules for the evolution of cooperation., Science 314 (2006) 1560-1563.

[18] L. Lehmann, L. Keller, S. West, D. Roze, Group selection and kin selection: two concepts but one process., Proc Nat Acad Sci USA 104 (2007) 6736-9.

[19] M. A. Nowak, C. E. Tarnita, E. O. Wilson, The evolution of eusociality., Nature 466 (2010) 1057-1062.

[20] P. Abbot et al, Inclusive fitness theory and eusociality., Nature 471 (7339) (2011) E1-4; author reply E9-10.

[21] M. van Baalen, D. A. Rand, The unit of selection in viscous populations and the evolution of altruism, J Theor Biol 193 (4) (1998) 631-648. 
[22] N. M. Shnerb, Y. Louzoun, E. Bettelheim, S. Solomon, The importance of being discrete: life always wins on the surface., Proceedings of the National Academy of Sciences of the United States of America 97 (19) (2000) 103224.

[23] M. Perc, Coherence resonance in a spatial prisoner's dilemma game, New Journal of Physics 8 (2) (2006) 22-22.

[24] M. Perc, A. Szolnoki, Social diversity and the promotion of cooperation in the spatial prisoner dilemna, Physical Review E 77 (1) (2008) 011904.

[25] Z. Patwa, L. M. Wahl, The fixation probability of beneficial mutations., J R Soc Interface 5 (2008) 1279-1289.

[26] W. J. Ewens, Mathematical Population Genetics, Springer-Verlag, 2004.

[27] G. Hardin, The tragedy of the commons., Science (New York, N.Y.) 162 (3859) (1968) 1243-8.

[28] T. Pfeiffer, S. Schuster, S. Bonhoeffer, Cooperation and competition in the evolution of ATP-producing pathways., Science (New York, N.Y.) 292 (5516) (2001) 504-7.

[29] R. C. MacLean, I. Gudelj, Resource competition and social conflict in experimental populations of yeast., Nature 441 (7092) (2006) 498-501.

[30] R. C. MacLean, The tragedy of the commons in microbial populations: insights from theoretical, comparative and experimental studies., Heredity 100 (5) (2008) 471-7.

[31] J.-U. Kreft, Biofilms promote altruism., Microbiology (Reading, England) 150 (Pt 8) (2004) 2751-60.

[32] P. B. Rainey, K. Rainey, Evolution of cooperation and conflict in experimental bacterial populations., Nature 425 (6953) (2003) 72-4.

[33] J. S. Chuang, O. Rivoire, S. Leibler, Simpson's paradox in a synthetic microbial system., Science (New York, N.Y.) 323 (5911) (2009) 272-5.

[34] B. Houchmandzadeh, M. Vallade, Selection for altruism through random drift in variable size populations., BMC Evol Biol 12 (2012) 61.

[35] A. Lambert, Probability of fixation under weak selection: A branching process unifying approach, Theor Popul Biol 69 (2006) 419-441.

[36] P. Moran, Statistical Processes of Evolutionary Theory, Clarendon Press, 1962.

[37] J. H. Gillespie, Natural selection for within-generation variance in offspring number, Genetics 76 (1974) 601-606. 
[38] M. Kimura, On the probability of fixation of mutant genes in a population., Genetics 47 (1962) 713-719.

[39] S. N. Ethier, M. F. Norman, Error estimate for the diffusion approximation of the Wright-Fisher model., Proc Nat Acad Sci USA 74 (1977) 5096-5098.

[40] E. G. Ruby, Lessons from a cooperative, bacterial-animal association: the Vibrio fischeri-Euprymna scolopes light organ symbiosis., Annual review of microbiology 50 (1996) 591-624.

[41] A. Ross-Gillespie, A. Gardner, S. A. West, A. S. Griffin, Frequency dependence and cooperation: theory and a test with bacteria., The American naturalist 170 (3) (2007) 331-42.

[42] C. Taylor, D. Fudenberg, A. Sasaki, M. A. Nowak, Evolutionary game dynamics in finite populations., Bull Math Biol 66 (2004) 1621-44.

[43] P. D. Taylor, L. B. Jonker, Evolutionary stable strategies and game dynamics, Mathematical Biosciences 40 (1-2) (1978) 145-156.

[44] M. A. Nowak, A. Sasaki, C. Taylor, D. Fudenberg, Emergence of cooperation and evolutionary stability in finite populations., Nature 428 (2004) $646-50$.

[45] C. P. Roca, J. A. Cuesta, A. Sánchez, Evolutionary game theory: Temporal and spatial effects beyond replicator dynamics., Physics of life reviews 6 (2009) 208-49.

[46] A. T. Silva, J. Fontanari, Stochastic group selection model for the evolution of altruism, Physica A: Statistical Mechanics and its Applications 268 (1999) 257-268.

[47] L. Lehmann, F. Rousset, How life history and demography promote or inhibit the evolution of helping behaviours., Philosophical transactions of the Royal Society of London. Series B, Biological sciences 365 (1553) (2010) 2599-617.

[48] M. Perc, A. Szolnoki, Coevolutionary games-a mini review., Bio Systems 99 (2) (2010) 109-25.

[49] O. Hallatschek, Noise driven evolutionary waves., PLoS Comp Bio 7 (2011) e1002005. 